\title{
LA EVOLUCIÓN DE LAS PENSIONES DE JUBILACIÓN Y VIUDEDAD DESDE LA PERSPECTIVA DE GÉNERO
}

\author{
The Evolution of Retirement Pension \\ and Survival Allowance from a Gender Perspective
}

\author{
Eva María Blázquez Agudo \\ Profesora Titular de Derecho del Trabajo y de la Seguridad Social \\ Universidad Carlos III, Madrid
}

\section{ABSTRACT}

En general, las beneficiarias de las pensiones de viudedad son mujeres, mientras que los beneficiarios de las pensiones de jubilación son hombres. Esta situación obedece a un antiguo reparto de roles familiares en el que el hombre salia a trabajar fuera de casa, mientras la mujer se quedaba cuidando de la familia. Ahora la inserción de la mujer en el mercado laboral está introduciendo nuevos elementos que deben tenerse en cuenta en la regulación de las pensiones. Es preciso poner remedio a las pérdidas de cotizaciones provocadas por necesidades de conciliación y a la brecha salarial que sufren las mujeres, ya que ambas situaciones van a influir negativamente en la consecución de la pensión de jubilación futura. Por otra parte, la pensión de viudedad ha perdido su fundamento de proteger a las mujeres que se quedaban en el hogar cuidando de su familia, de modo que es preciso replantearse su contenido.

Palabras clave: pensión de jubilación, pensión de viudedad, género, derecho a la seguridad social. 
Generally, the beneficiaries of survival allowances are women, whereas the beneficiaries of retirement pensions are men. This situation's reason is a very old division of roles: men worked and women took care of their family. Nowadays, the insertion of women into the labour market has introduced new elements, which must be considered by the pension law. It is necessary to remedy the negative consequences of the conciliation between family and working life in the contributions of social security and the salary gap, because both are going to have influences in the access to future pensions. On the other hand, the survival allowance has lost its essence: the protection of women, who were at home to care their family. Then, it is the moment to rethink about its regulation.

Keywords: survival allowance, retirement pension, gender, social security rights. 


\section{SUMARIO}

1. Introducción. 2. La realidad de las pensiones en cifras. 2.1. La brecha en la cuantía de las pensiones. 2.2. Los efectos del complemento por mínimos. 2.3. Las pensiones no contributivas. 3. La pensión de jubilación desde la perspectiva de género. 3.1. Los efectos de la exigencia de mayores cotizaciones en las últimas reformas 3.2. Los remedios legales para contrarrestar los efectos en las cotizaciones de los cuidados familiares. 3.2.1. El reconocimiento de cotizaciones ficticias vinculadas a la maternidad y al cuidado. 3.2.2. La interpretación humanizadora del período de carencia específica 3.2.3. La aplicación de reglas atenuadoras de los efectos de los contratos a tiempo parcial. 3.3. El complemento por maternidad. 4. La pensión de viudedad desde la perspectiva de género. 4.1. La necesaria adaptación de la pensión de viudedad a la realidad actual. 4.2. La doble naturaleza de la pensión: contributiva para el causante y cuasi-asistencial para el beneficiario. 4.3. Dos opciones de cambio ofrecidas para conseguir el equilibrio financiero. 4.3.1. El posible cambio del tipo de financiación. 4.3.2. La incompatibilidad de la pensión con salario y otras prestaciones sustitutivas. 4.4. Hacia el necesario reconocimiento de derechos propios a los ciudadanos.

\section{Introducción}

En la actualidad, se realizan estudios en materia de pensiones que versan sobre la necesidad de incluir cambios legislativos en el modelo actual a los efectos de garantizar la sostenibilidad financiera del sistema. Se insiste en el análisis de los requisitos de acceso y la necesidad de modificarlos a los efectos de moldearlos de acuerdo con estas cuestiones. Pero, en este panorama se echan de menos estudios sobre estas materias desde el punto de vista de género.

Hombres y mujeres no han tenido, ni tienen, las mismas condiciones sociales y laborales, esta realidad se refleja en el acceso a las pensiones, tanto de jubilación como de viudedad. Es por esto por lo que un cambio en el modelo pasa por el estudio de los datos desagregados de los beneficiarios de las prestaciones, pero también por el de las condiciones laborales de los actuales trabajadores con el fin de poder adaptar la regulación de las pensiones a sus realidades.

Como luego se pondrá en evidencia a través de los datos estadísticos, los beneficiarios de la pensión de jubilación son mayoritariamente hombres y, en el caso de la pensión de viudedad, mujeres. Esta situación tiene su origen en el reparto de roles en las familias hace unas décadas. La generación, que ahora tiene 
edades superiores a la legal de jubilación, repartía las labores de forma que uno trabajaba y generaba pensiones, y el otro quedaba en casa, cuidando de la familia. Y dicha realidad tiene incidencia en las pensiones que ahora se disfrutan.

No obstante, una realidad distinta como la masiva incorporación de la mujer al mercado laboral en los últimos ańos hace preciso volverse a replantear las pensiones, poniendo en evidencia cómo este factor va a afectar a las prestaciones futuras. En este contexto, es fundamentar valorar la especial situación de las trabajadoras frente a las responsabilidades familiares, así como las consecuencias de la brecha salarial en las bases reguladoras de las pensiones.

En este estudio se quieren valorar ambas situaciones. Por un lado, cuáles son los principales problemas a que se enfrentas las mujeres al acceder o disfrutar de las pensiones de jubilación y viudedad en la actualidad. Pero, asimismo, sin olvidar cómo el cambio social debe ponerse en valor a los efectos de ir modificando la legislación para adaptarse a estos.

Con este objetivo se plantea este trabajo. En primer lugar, se va a exponer la realidad de las pensiones actuales que disfrutan las mujeres desde el análisis de los datos estadísticos. Con posterioridad, se pasará a examinar ambas pensiones, jubilación y viudedad, desde la perspectiva de género, partiendo de la actualidad, pero con el ánimo de aportar ciertas pinceladas sobre lo que desde la opinión de la autora debería ser el futuro de ambas.

\section{La realidad de las pensiones en cifras}

Los beneficiarios de las pensiones de jubilación son especialmente los hombres y las beneficiarias de las pensiones de viudedad las mujeres. Esta afirmación es fácil de contrastar con los datos estadísticos (MESS, 2017). Respecto a la pensión de jubilación, son 3.647.914 hombres frente a 2.195.852 mujeres. Estos resultados tienen claramente su origen en la realidad social anterior, donde las mujeres mayoritariamente no trabajaban fuera de casa. Son principalmente los trabajadores que nacieron en los ańos treinta, cuarenta y cincuenta, los que se benefician de la jubilación y, es por esto, que es lógico que los hombres, que en su mayoría trabajaban y, por tanto, cotizaban, lleguen a la pensión de jubilación. No obstante, es posible que en los próximos años se vaya acercando el número de trabajadores y trabajadoras pensionistas, debido a los efectos de la incorporación masiva de las mujeres en las últimas décadas.

\subsection{La brecha en la cuantía de las pensiones}

No obstante, existe una brecha aún más importante y preocupante que la del número de pensionistas hombres y mujeres, puesto que las cifras irán modi- 
ficándose y convergiendo, en cierta medida, con el paso de tiempo: es el hecho del importe de la pensión. Así, la pensión media de jubilación de los hombres es de 1.236,34 euros frente a los 785,18 euros de las mujeres. Como se ve, la diferencia es muy significativa y es esperable que se mantenga en el futuro, dado que como las mujeres siguen percibiendo salarios más bajos, cotizan menos y, consecuentemente, acceden a prestaciones en cuantías inferiores.

La conclusión es la contraria en el análisis de la pensión de viudedad. Hay 2.180.835 mujeres percibiendo la pensión frente a 180.724 hombres. Aquí el razonamiento de los datos es el contrario. Como fueron pocas las mujeres que trabajaron y cotizaron, han causado menos pensiones de viudedad a su fallecimiento. Además, por otra parte, también las mujeres tienen una expectativa de vida superior que los hombres por lo que, en general, hay más viudas que viudos, con independencia de que trabajasen o no.

La pensión media de viudedad es de 661,19 euros para las mujeres, mientras que para los hombres es de 486,20 euros. De nuevo, la mayor cotización de los trabajadores hace que se causen pensiones con cuantías más altas, esta vez en favor de las mujeres.

En definitiva, si se suman las pensiones de viudedad y de jubilación que perciben las mujeres, se estará incluyendo en el colectivo a casi $4.400 .000 \mathrm{mu}$ jeres, cuyas retribuciones medias son de alrededor de 700 euros. En contraposición con el colectivo de los hombres, cuyas pensiones medias totales son de casi 1.300 euros. Podría alegarse que hay 180.480 hombres recibiendo una pensión de viudedad en bastante menor cuantía que cualquiera de los colectivos analizados, no obstante, es fácil deducir que estos beneficiarios en la mayoría de los casos tendrán sus pensiones propias de jubilación, dado que suelen acceder a pensiones de jubilación o, en su caso, realizar una actividad laboral retribuida. En el caso de las mujeres que compatibilicen las pensiones de jubilación y viudedad, un grupo mucho más reducido, si se suma la media del importe de ambas pensiones, el importe obtenido se igualará al que reciben de media los hombres solo como pensión de jubilación.

Así, solo las mujeres que compatibilizan la pensión de jubilación y de viudedad podrán obtener cuantías medias similares a las que alcanzan los hombres con el importe medio de la pensión de jubilación. En definitiva, se podrá mantener que es difícil concluir la suficiencia de las pensiones que perciben las mujeres, elemento imprescindible, proclamada por el propio artículo 41 de la Constitución que regula el derecho a la Seguridad Social.

\subsection{Los efectos del complemento por mínimos}

Para acceder al complemento por mínimos se exige que el beneficiario demuestre su carencia de rentas, aunque se trate de una pensión contributiva. Se 
exige que sus ingresos (salariales, de capital o de origen prestacional) sean inferiores a una cantidad cierta que será determinada anualmente en la Ley de Presupuestos del Estado y como no tiene carácter consolidable cada ejercicio deberán demostrarse de nuevo estas circunstancias de insuficiencia. La Ley 24/1997, de 15 de julio, de Consolidación y Racionalización del sistema de la Seguridad Social, proclamó la naturaleza asistencial de dichos complementos, dado que se señalaba que debían ser financiados a través de impuestos, tal y como que se recoge en la propia LGSS, aunque no fue hasta la Ley de Presupuestos de 2014 donde este cambio en la financiación del complemento desde las cotizaciones a los impuestos se hizo efectivo.

La determinación de la cuantía de la pensión mínima depende de dos variantes: la edad del pensionista y la existencia de cónyuge a cargo. De la combinación de estos dos elementos, se establecen cuantías distintas para los complementos por mínimos. En el caso de que el pensionista tuviese un cónyuge a su cargo, se le asegura una pensión superior. En 2017, los relativos a la pensión de viudedad son concretamente: a) para los mayores de 65 años o beneficiarios con discapacidad, 637,70 euros; b) para los beneficiarios de entre 60 y 64 años, 596,50 euros; y para los menores de 60 ańos es de 482,90 euros. Este importe se eleva en los supuestos que se demuestra la carencia de rentas y las cargas familiares hasta 737,60 euros. Frente a estas cuantías, los mínimos para la pensión de jubilación son: para los mayores de 65 años, 786,90 euros con cónyuge a cargo; 637,70 euros, en hogares unipersonales; y 605,10 euros en caso de tener cónyuge, pero no a cargo. Se iguala la cuantía de la pensión mínima de viudedad general con la que recibirán los pensionistas de jubilación en hogares unipersonales.

Del análisis de las cuantías, se puede observar cómo estos complementos por mínimos en la pensión de jubilación, que se incrementan en caso de cónyuge a cargo, continúan reflejando el reparto clásico de roles en el hogar. Así, son los hombres los que perciben especialmente este complemento por sus esposas a cargo; mientras que son menos las mujeres que lo reciben, puesto que, en la mayoría de los supuestos sus esposos tendrán sus pensiones propias y no se encontrarán a cargo de ellas.

No obstante, lo que más afecta a la brecha prestacional, y que influye en la feminización de la pobreza de las pensionistas, es la cuantía de los complementos por mínimos en las pensiones del SOVI. Aquí la pensión mínima es de 408,10 euros. A 1 de septiembre de 2017 hay un total de 25.813 pensiones de viudedad del SOVI que perciben mujeres, frente a solo 925, donde el beneficiario es el hombre. En este caso, la pensión media de las mujeres es de 380 euros. Estas pensiones son insuficientes y contribuye al aumento de la pobreza femenina, alejándolas de la autonomía económica. 


\subsection{Las pensiones no contributivas}

Si se pone ahora la vista en el ámbito de las pensiones no contributivas propias, al revés que en las contributivas, se puede afirmar que las mujeres son el grupo que obtiene más prestaciones de acuerdo con los datos estadísticos (IMSERSO, 2016). En general, el 66,42\% de los pensionistas son mujeres (301.682) frente a un 33,458\% de hombres (152.519). Esta diferencia se hace aún más aguda cuando se toman solo los datos de la pensión de jubilación no contributiva. En este caso, el $77,71 \%$ son mujeres (154.171) frente a 22,29\% de hombres (44.232).

Parece que es fácil deducir que la razón para esta diferencia es que generalmente las mujeres tienen menos probabilidades de acceder a las cotizaciones mínimas para acceder a las pensiones de jubilación (15 años). Así, en unos casos no se ha trabajado; se ha trabajado durante cortos períodos de tiempo, en muchas ocasiones debido a cuestiones relacionadas con la atención de las responsabilidades familiares; o en otros, aunque se cotizó el mínimo exigido, no se ha hecho durante los últimos años, de modo que no es posible cumplir el requisito de la cotización específica (al menos, 2 años de cotización deben haberse realizado en los últimos 15). Solo un $23 \%$ de los pensionistas no contributivos son hombres, lo que parece que significa que mayoritariamente los trabajadores acceden al ámbito contributivo, ya que cotizan durante más años y pueden disfrutar de la protección del nivel contributivo.

Cuando las mujeres no pueden acceder a la pensión de jubilación y tampoco tienen otras rentas, por ejemplo, generadas por la pensión de viudedad, la única opción a partir de los 65 años es solicitar una pensión no contributiva de jubilación. Y, no hay que olvidar de nuevo los importes de estas prestaciones. La básica es de 368,90 euros al mes que puede elevarse según quién conviva en el hogar con el pensionista. Otra vez podrá volverse a poner en evidencia la situación de las mujeres pensionistas que, en su conjunto, tanto en el ámbito contributivo como asistencial, perciben menos ingresos que los hombres.

\section{La pensión de jubilación desde la perspectiva de género}

Como ya se ha señalado, existen menos beneficiarias de las pensiones de jubilación que beneficiarios, aunque es esperable que cada vez haya más, debido a los efectos de la incorporación de la mujer al mercado laboral. Si bien es verdad que el incremento se producirá naturalmente, debido a la evolución social, lo que más preocupa en este tema es el importe medio de la pensión, que como ya se ha señalado, es bastante inferior cuando se compara entre hombres y mujeres. 
Obviamente es fácil deducir cuáles son los elementos que influyen en esta diferencia. En concreto, hay dos factores relevantes. En primer lugar, la brecha salarial entre trabajadores y trabajadoras, y en segundo los efectos de la conciliación de la vida familiar y laboral en el desarrollo de las carreras profesionales de las mujeres.

Respecto a la brecha salarial, son suficientemente ilustrativos los datos de la Unión Europea, donde se pone de manifiesto que el 2015 la brecha salarial entre trabajadores y trabajadoras se encontraba de media en el 16,3\%, elevándose al 16,8 \% en la zona euro. En España se situaba cercana al 15\% (Gender pay gap statistics, marzo 2017).

En la mayoría de los supuestos las trabajadoras son quienes en mayor medida asumen las responsabilidades familiares (INE, 2010). Es un hecho constatado que las mujeres soportan de promedio mayor carga familiar, sobre todo en los tramos centrales de la vida laboral (de 35 a 50 años), de modo que parece lógico que sean ellas las afectadas en las cotizaciones que se precisan para acceder o calcular la pensión de jubilación.

La asistencia familiar es frecuentemente asumida por las mujeres, lo que trae importantes consecuencias en la incorporación de la mujer al mundo laboral y, consecuentemente, en el acceso a la protección social. En unas ocasiones, porque no ejercen actividad laboral (IMSERSO, 1995), en otras porque abandonan temporalmente su actividad para dedicarse a estas actividades, lo que provoca lagunas de cotización (Durán, 2006: 59). Otro importante factor que está mermando las carreras de cotización de las mujeres es la utilización masiva de la reducción de jornada por cuidado de familiares dado que, al aminorar paralelamente su salario, asimismo aminora sus cotizaciones a la Seguridad Social.

\subsection{Los efectos de la exigencia de mayores cotizaciones en las últimas reformas}

En las últimas modificaciones de la pensión de jubilación se han introducido unas mayores exigencias para acceder a los beneficios. Por otro lado, para alcanzar las cuantías más altas se demanda un mayor número de años cotizados. Estas reformas van a producir un efecto especialmente pernicioso en las mujeres, ya que ellas tienen más dificultad en acreditar cotizaciones a través de su vida laboral, debido a la influencia de factores relacionados con la maternidad.

En primer lugar, progresivamente se van a valorar más años de cotización del sujeto (hasta 300 meses en 2020) para determinar la cuantía de la pensión de jubilación. Es complicado que las mujeres puedan aportar 25 años de cotización de forma continua. A las dificultades generales de todos los trabajadores debido a la alta tasa de desempleo de la larga duración, donde no se consiguen 
cotizaciones, se tendrán que añadir ciertas peculiaridades que les afectan en especial a las mujeres, que interrumpen o reducen su carrera por motivos de cuidado familiar. Si la edad legal de jubilación va a ser 67 años (o 65 años para las largas carreras de cotización, lo cual es complicado en el caso de las mujeres), esto significará que se tendrán en cuanta las bases de cotizaciones desde los 43 años. En este período es probable que se encuentren vacíos de contribución por dos motivos: en primer lugar, por efecto la atención de los hijos, pero también por el cuidado de sus ascendientes en los últimos años.

Así, como es fácil deducir, en muchas ocasiones existirán períodos de cotización en blanco en esos 300 meses a valorar para determinar la cuantía de la pensión. Tampoco ayudan a resolver este problema las nuevas reglas de integración de lagunas, que solo complementan con el $100 \%$ de la base mínima de cotización durante 48 meses, mientras que el resto se integrarán con el $50 \%$ de dicha base. Es decir, que las mujeres que tengan más de 48 meses sin cotización entre esos 300, se les integrará el resto con un $50 \%$, lo que significara una merca significativa en su base reguladora. Todavía será peor la situación de la trabajadora autónoma o que desarrolle su actividad en el sistema especial de empleadas del hogar, que ni siquiera se les ofrecerá la opción de completar sus bases, en los meses no cotizados.

Por otro lado, cada vez se demanda más número de años de contribución a los efectos de aplicar el $100 \%$ a la base reguladora (hasta 37 años en 2027). La dificultad de las mujeres en el mercado laboral llevará sin duda a reducir las opciones de alcanzar las pensiones máximas debido a la irregularidad de sus carreras profesionales.

Todo esto sumado a otros factores que van a afectar a todos los trabajadores, tales como los elementos sancionadores en el caso de jubilaciones anticipadas, en muchas ocasiones involuntarias; la aplicación del factor de sostenibilidad a partir de 2019 (un instrumento automático que vincula importe de pensión a evolución esperanza vida pensionistas, ajustando la cuantía en el momento del cálculo de acuerdo con tablas de mortalidad que se revisarán periódicamente); o el nuevo modelo de cálculo de la revalorización de pensiones, llevará a que el importe de la pensión de jubilación de las mujeres sea especialmente exiguo y, en muchas ocasiones, precise de ser complementado a mínimos, de forma que solo la garantía de un importe suficiente en este ámbito podrá asegurar la subsistencia de las pensionistas.

\subsection{Los remedios legales para contrarrestar los efectos en las cotizaciones de los cuidados familiares}

En los últimos tiempos, se han regulado ciertos remedios para evitar los efectos perniciosos de la dedicación al cuidado de los familiares, que sufren las trabajadoras, en el acceso a la pensión de jubilación. 
En concreto, se trata del reconocimiento de cotizaciones ficticias relacionadas con la maternidad con el fin de colaborar en el aumento del período cotizado a los efectos de facilitar el cumplimiento de las exigencias mínimas de carencia; la interpretación humanitaria del cumplimiento del período de carencia específica (la acreditación de 2 años de cotización en los últimos 15 antes del hecho causante); la aplicación de reglas específicas de las exigencias del período de carencia de acceso a la pensión de los trabajadores a tiempo parcial (en su mayoría mujeres); y por último, el complemento por maternidad que se reconoce tanto en la pensión de jubilación como en la de viudedad e incapacidad permanente.

Ya antes de entrar en su análisis hay que poner de manifiesto que se entiende que estos remedios son solo parches precisos para ayudar a las trabajadoras en el acceso a la pensión de jubilación. Pero, que realmente, su regulación debería ser pasajera mientras se trabaja en el ámbito de la inserción de la mujer en el mercado laboral. Solo con la equiparación en el trabajo de los trabajadores y trabajadoras, especialmente en el salario, se pueden evitar las dificultades de acceso a la protección social de las mujeres. El mejor remedio a esta situación debería ser la flexibilización del mercado (tanto en tiempo como en lugar) con el fin de que colaborar en la corresponsabilidad y conseguir la igualdad salarial y de cotizaciones, que será lo único que garantice pensiones de jubilación adecuadas en el futuro.

\subsubsection{El reconocimiento de cotizaciones ficticias vinculadas a la maternidady al cuidado}

Además del natural reconocimiento del período de cotizaciones efectivamente realizadas en situación de baja por incapacidad temporal producida por el embarazo, maternidad, paternidad, riesgo durante el embarazo o durante la lactancia natural, se conceden ciertos períodos asimilados a cotizados. En concreto, se reconocen otras cotizaciones durante los periodos de maternidad o paternidad subsistente a la fecha de extinción de la relación laboral o que se inicie durante la percepción de la prestación por desempleo, los periodos de excedencia para cuidado de hijo o menor acogido; los períodos de cotización asimilados a parto; y los períodos considerados como cotizados a causa de la violencia de género, durante el periodo de suspensión del contrato de trabajo, en razón del derecho de protección ante la situación de violencia de género (con un máximo de 6 meses, salvo que el juez haya prorrogado el tiempo de suspensión con un máximo de 18).

A continuación, se analizan alguno de ellos. El período de cotización por parto se reconoce exclusivamente a las madres, cuando no hayan contribuido a la Seguridad Social en el tiempo posterior al parto. Por tanto, no se reconoce a las trabajadoras que hayan suspendido su contrato de trabajo por maternidad y 
hayan percibido la prestación de maternidad, puesto que durante este tiempo ellas sí han estado cotizando por la base que resulte de hacer la media entre las bases de cotización en los últimos 180 días. Así, aquellas que no trabajaban o por no acreditar el período de carencia mínimo accedieron al subsidio asistencial por maternidad, podrán beneficiarse del reconocimiento de 112 días de cotización por cada parto de un solo hijo, a los que se sumará 14 días más por cada hijo a partir del segundo en caso de parto múltiple.

Varias cosas hay que poner de manifiesto en relación con este remedio. En primer lugar, no iguala a los hijos naturales con los adoptados, lo cual parece que vulnera el artículo 39 de la Constitución Española que reconoce la total equiparación de los hijos naturales y los adoptivos. Por otra parte, si bien es verdad que el supuesto más común es que sea la madre la que se encuentre en esta situación de desprotección en ese momento del nacimiento de sus hijos, también es posible para los padres. Si se trata de ir reconociendo derechos individuales a los trabajadores con independencia de su género, esta regulación denegaría esta opción para los hombres. Otra cosa es que, en caso de que coincidiesen ambos progenitores en la situación, se reconociese el derecho a la madre preferentemente, como ocurre en el reconocimiento de cotizaciones por períodos de cuidado, tal y como se analizará a continuación.

El siguiente reconocimiento de períodos ficticios de cotización se hace en caso de abandono de la carrera de cotización por cuidado de hijo. En este supuesto, se reconocen días de cotización por cada hijo tanto a los efectos de determinar la edad de jubilación como para el cálculo de su cuantía. En la actualidad el reconocimiento de días se encuentra en un período transitorio que terminará en 2019, a partir de cuya fecha se computan 270 días por cada hijo cuidado, con independencia que hayan nacido o hayan sido adoptados, al mismo tiempo.

Se reconocen a las trabajadoras, pero también a los trabajadores que abandonan su actividad para dedicarse al cuidado del menor. Se entiende que se ha producido la interrupción de la cotización por este motivo cuando se haya extinguido la relación laboral o se haya finalizado el cobro de prestación de desempleo entre los 9 meses anteriores al nacimiento del hijo y los 6 ańos posteriores a este hecho. Si ambos progenitores cumplen estas condiciones, el reconocimiento privilegia a la madre.

Este beneficio salva las dos críticas que se hacen al reconocimiento de cotizaciones ficticias por razón de parto, ya que se concede tanto por cuidado de hijo natural como adoptivo, así como se reconoce a las trabajadoras como a los trabajadores.

Por último, aunque no se trata de reconocimiento de cotizaciones ficticias como tal, hay otra figura en este ámbito que también es un remedio a las reduc- 
ciones de cotizaciones. Así, en vez de computar la cotización a tiempo parcial de acuerdo con la jornada que realice el empleado con jornada reducida se hace de forma completa. Así, se reconoce como cotizado al $100 \%$ los períodos de reducción de jornada por cuidado de hijo menor de 12 ańos, aunque solo durante los dos primeros años. Si la reducción es por cuidado de otros familiares, este derecho solo se concede durante un año. En la misma línea, se entienden cotizados de idéntica manera durante todo el tiempo que dure a la reducción de jornada por cuidado de menor afectado de cáncer u otra enfermedad grave. Igualmente, se concede un tratamiento especial a los períodos de excedencia por cuidado de menor o familiares, precedidos de una reducción de jornada por cuidado de hijo o familiar.

Aunque estos derechos son reconocidos tanto a los trabajadores como a las trabajadoras, son ellas las que generalmente utilizan estas reducciones. Así, una de cada cinco mujeres redujo su jornada laboral por motivos de cuidado de sus hijos y una de cada tres de estas mujeres, que tomó excedencia, lo hizo por un periodo de seis a 12 meses. Además, el mismo estudio señaló que de las 685.300 personas que sí redujeron su horario laboral, el 85,1\% fueron mujeres y el $14,9 \%$ varones (INE, 2011). De forma que queda probado que el problema de la reducción de jornada laboral en los efectos de aminorar salario y consecuentemente cotización afecta especialmente a las trabajadoras. De modo que los remedios antes explicados, serán medidas dirigidas indirectamente a las mujeres.

Como se ha señalado, es adecuado que se regulen remedios que traten de aminorar los efectos perniciosos de esta situación en la futura pensión de jubilación. Pero, en el concreto caso de la reducción de la jornada por motivos de cuidado de hijo menor de 12 años, no se debe olvidar que el remedio solo se aplica durante dos de estos años, de los casi doce que se puede tener reducida la jornada por este motivo. Con lo cual es previsible que las consecuencias en una mujer, por ejemplo, que tenga dos hijos y reduzca la jornada por cuidado de ambos, puedan ser casi catastróficas en su carrera de cotizaciones.

Una opción para resolver estas consecuencias podría ser extender este beneficio a todo el tiempo que dura la situación de reducción de la jornada por cuidado por hijos, al igual que ocurre en el supuesto de cuidado de hijo enfermo, no obstante, la aminoración de la protección social no es la única consecuencia de dicha reducción. De modo que la elección de la extensión de este beneficio podría tener indirectamente consecuencias derivadas aún más graves que los efectos actuales en las carreras de cotización.

Asimismo, la reducción de la jornada por cuidado de hijos produce la pérdida de las posibilidades de promoción y, en muchos casos, de inserción laboral, dado que en las empresas se conoce bien esta opción de la trabajadora y dados los efectos negativos de organización que les puede causar, es posible que se ali- 
mente la tendencia a contratar preferentemente a los hombres. De nuevo, desde aquí debe ponerse en evidencia que si bien es positivo que se regulen remedios transitorios que soluciones los problemas de cotización de las trabajadoras, la mejor solución sigue siendo la flexibilización del tiempo y lugar de trabajo con el objeto de evitar la necesidad de utilizar las reducciones de jornada o las excedencias como los únicos mecanismos que abren la posibilidad de conciliar la vida familiar y laboral.

Dentro del contexto de estos remedios para las trabajadoras en el ámbito de la cotización, se puede presentar la opción del derecho alemán que contempla el traspaso de cuotas desde el consorte trabajador al cónyuge que no ha realizado actividad laboral por dedicarse al cuidado directo de la familia, lo que lleva a este último a adquirir las prestaciones de vejez por derecho propio. Es decir, se apuesta por el reparto de cotizaciones a los efectos de que cada trabajador y trabajadora pueda acceder a su pensión propia de jubilación. Además, esta fórmula no rompe el principio de contributividad que, como luego se analizará, sí lo hace la concesión de dos pensiones causadas por un mismo sujeto.

De esta forma, si se han repartido las funciones familiares, ambas partes quedarán beneficiadas por las contribuciones hechas a la Seguridad Social, con independencia de quien las haya realizado. De hecho, los nacidos a partir de cierta fecha pueden elegir entre la pensión de viudedad o por el reparto equitativo de las pensiones de jubilación entre ambos cónyuges en vida de los mismos. No sólo se reparten los importes de las pensiones, sino que los periodos de cotización les sirven a ambos cónyuges para cubrir el periodo de cotización general que se exige para tener derecho a las distintas pensiones de jubilación. En la misma línea, en Colombia, se reconoce una pensión familiar que consiste en que las parejas podrán sumar sus semanas de cotización para completar el tiempo requerido y de esta manera recibir una pensión conjunta.

\subsubsection{La interpretación humanizadora del periodo de carencia específica}

Para acceder a la pensión de jubilación se exige tener cotizados, al menos, 15 años. Pero, además, existe una condición añadida que es la necesidad de acreditar que 2 de esos años se hayan conseguido en los 15 últimos años. Puede ser usual que una mujer tenga los 15 años de cotización a lo largo de su vida laboral, pero que, como abandonó la actividad laboral para dedicarse al cuidado de su familia, no pueda acreditar este último requisito.

La jurisprudencia ha aplicado una interpretación humanizadora a este requisito, que consiste en que cuando una persona no pudo cotizar por causa ajena a su voluntad durante un cierto período, se coloca dicho lapso entre paréntesis sin que se tenga en consideración en la determinación del periodo de los quince últimos ańos antes del hecho causante donde se tienen que acredi- 
$\operatorname{tar} 2$ ańos. Esto ayuda a todos los trabajadores a acceder a la pensión de jubilación, pero especialmente a las mujeres que se van a encontrar seguramente más en esta situación.

Por ejemplo, el Tribunal Supremo ha considerado como situación en que debe establecerse el paréntesis: principalmente, la situación de paro involuntario no subsidiado siempre que exista una permanente inscripción como demandante de empleo; la enfermedad grave; o el acceso a la pensión no contributiva de invalidez.

\subsubsection{La aplicación de reglas atenuadoras de los efectos de los contratos a tiempo parcial}

La STC 61/2013, de 14 de marzo declaró que la regulación sobre la cotización en los contratos a tiempo parcial vulneraba el principio de igualdad, dado que hay muchas más mujeres que hombres desarrollando una actividad laboral a tiempo parcial, por lo que va a ser este colectivo el afectado directamente por la reglamentación inadecuada de la protección de los contratos a tiempo parcial. Posteriormente, fue la Ley 1/2014, de 28 de febrero, la que modificó la normativa para evitar esta discriminación y estableció algunos remedios para atenuar las consecuencias en las pensiones de este tipo de actividad.

La declaración del Tribunal Constitucional queda avalada por la realidad de los datos. Así, según cifras publicadas por el INE correspondientes a los meses de enero a septiembre de 2017 existen un total de 3.29.760 de mujeres con un contrato parcial frente a 2.373.900 de hombres.

Las cifras son más elocuentes aún si estos resultados se comparan con el número de contratos a tiempo completo. Hay 3.687.000 trabajadoras a tiempo completo. De modo que el número de contratos a tiempo parcial y a tiempo completo de las mujeres es bastante similar. Mientras que en el caso de los hombres con contrato a tiempo completo es de 6.640.300. Es decir, los trabajadores a tiempo completo casi triplican el número de trabajadores a tiempo parcial. En conclusión, todos los efectos negativos que se puedan provocar en el ámbito de los contratos parciales con el fin de acceder a las pensiones de jubilación tendrán más impacto en el colectivo de las mujeres. Y, todo esto, incluso sin analizar la voluntariedad en la decisión de trabajar en esta modalidad, cuestión que subyace en este problema, donde se intuye que el número elevado de mujeres a tiempo parcial se debe a dos elementos fundamentales: uno la necesidad de conciliar con su vida familiar y otra la dificultad del acceso a contratos a tiempo completo.

En concreto, en la Ley de 2014 se regularon elementos que atenuaron los efectos negativos de la cotización a tiempo parcial en el período de carencia de acceso a las prestaciones, y en la forma de cálculo de las bases reguladoras. 
Respecto al período de carencia, ya no es preciso acreditar los 15 años de cotización en caso de haber trabajado a tiempo parcial para acceder a la pensión de jubilación. Se aplican unas reglas atenuadoras de estas circunstancias dependiendo del porcentaje del tiempo trabajado en comparación con el mismo trabajo a tiempo completo; y de la duración del trabajo a tiempo parcial en comparación con el tiempo total en el que se ha desarrollado actividad laboral por el beneficiario. En relación con la base reguladora se aplica de forma general un coeficiente del 1,5 para atenuar las consecuencias de las ínfimas bases de cotización que suelen presentar los trabajadores a tiempo parcial debido a sus bajos salarios.

En conclusión, con estas matizaciones se facilita a las mujeres que desarrollan una actividad laboral a tiempo parcial el acceso a la pensión de jubilación. No obstante, una vez más hay que poner en evidencia que los problemas planteados quizás pueden tener una base en el propio funcionamiento del mercado laboral que en muchos casos dificulta el acceso de la mujer al mercado de trabajo a tiempo completo. Así, la aplicación de estos remedios debería ser algo residual y lo que habría que aplicar son medidas para evitar la contratación masiva de las mujeres a tiempo parcial, en caso de no ser una opción deseada.

\subsection{El complemento por maternidad}

Recientemente se ha regulado un complemento por maternidad cuyo objetivo es premiar a las mujeres por su aportación demográfica a la Seguridad Social, esto es, por traer nuevos cotizantes que se encargarán de contribuir al sistema en el futuro.

Se concede para complementar a tres tipos de pensiones: la de jubilación, la de incapacidad permanente y la de viudedad. Pero, este beneficio no se ha reconocido de forma generalizada para todas las pensiones, sino que se limita el reconocimiento a las causadas a partir de 2016.

Las beneficiarias no son todas las madres, sino solo aquellas que hayan tenido, al menos, dos hijos, con independencia de que sean naturales o adoptivos. La cuantía de la pensión correspondiente se eleva porcentualmente dependiendo del número de hijos. Así, el incremento es de un $5 \%$ de la base reguladora que se haya empleado para determinar la pensión en cuestión, cuando tenga dos hijos; si tiene tres podrá beneficiarse de hasta un $10 \%$; y a partir de cuatro se eleva hasta un $15 \%$. Obviamente para evitar que no tenga efectos en aquellas mujeres que tengan las bases más altas, se concede, en su caso, más allá del tope máximo de pensiones. De forma que si se tiene topada la pensión a la máxima, a esta cuantía se le sumaría luego el complemento de maternidad.

Varias críticas se pueden verter este complemento. Su fundamento principal no puede ser solo el premio a la aportación de hijos a la Seguridad Social, 
puesto que en este caso se tendría que haber concedido también a los hombres que sean padres. Parece más bien una forma de atenuar las consecuencias negativas que la maternidad puede provocar en los salarios, en las bases de cotización y, consecuentemente, en las pensiones, obviamente cuando se trata de complementar las pensiones de jubilación e incapacidad permanente. En el caso de la pensión de viudedad, no puede ser otra que el reconocimiento del cuidado de la familia.

Por otro parte, tampoco se entiende que solo se reconozca a las mujeres que tienen más de dos hijos. En un contexto de baja natalidad parece que lo más adecuado habría sido extender el reconocimiento a las madres de un solo hijo.

Para finalizar parece más conveniente regular medidas que ayuden a criar a los hijos y que, por tanto, tengan efectos en el desarrollo de las carreras profesionales de las mujeres por sí mismas que medidas que busquen premiar a las madres a posteriori. Sería interesante establecer políticas familiares que, además de reconocer la aportación a la Seguridad Social, también colaborasen en los costes aparejados a los hijos, de este modo, asimismo, se podría tener un efecto de cooperar en que el número de hijos se adapte a los deseos de los ciudadanos, sin necesidad de premiar por haberlo hecho sin ninguna ayuda.

\section{La pensión de viudedad desde la perspectiva de género}

La pensión de viudedad se regula en la Orden de 13 de febrero de 1967, por la que se establecen las normas para la aplicación y desarrollo de las prestaciones de muerte y supervivencia del régimen general de la Seguridad Social. Su ordenación se basa en la situación social de hace 40 años, donde la mujer se quedaba al cuidado de los hijos y el marido salía de casa para obtener un salario para mantener a todo el grupo familiar. Con este origen, no es de extrañar que los hombres no pudiesen acceder a esta prestación. No fue hasta la Ley 26/1990, de 20 de diciembre, por la que se establece prestaciones no contributivas de la Seguridad Social, cuando se eliminó cualquier tipo de diferencia de trato en la pensión de viudedad por razón de sexo, sustituyendo el término «viuda» por el de «cónyuge», dado que el Tribunal Constitucional reconoció la discriminación por razón de sexo, en este caso, a los hombres (STC 103/1983, 22 de noviembre). Con anterioridad solo se reconocía el derecho cuando los beneficiarios probasen su incapacidad para el trabajo y su dependencia de su esposa fallecida, condiciones que no se demandaban a las viudas, ya que se presumía que ellas sí cumplían estas condiciones.

De acuerdo con las notas descritas, las pensiones de viudedad se regularon en el ámbito contributivo, pero, dependiendo de las cotizaciones del causante y no del beneficiario. Es decir, que como se presumía que era el hombre quien 
trabajaba por toda la familia, cotizaba para causar dos pensiones, una la suya propia y otra la de su cónyuge.

A lo largo de estos 40 años, se han introducido modificaciones legislativas y matizaciones jurisprudenciales con el fin de adaptar la pensión de viudedad a la marcha de los tiempos. Así, por ejemplo, cuando en 1981 se aprobó el divorcio en España, se reconoció la posibilidad de que no solo accedieran los cónyuges a la pensión de viudedad, sino también los excónyuges. Posteriormente, cuando aumentó el número de parejas de hecho, se incluyó igualmente a estas en el ámbito subjetivo de la pensión, siempre cumpliendo ciertos requisitos que demuestren su estabilidad. Pero, todavía queda un paso más: su ajuste a la inserción de la mujer en el mercado laboral y a la superación del reparto de roles mujer-hombres.

\subsection{La necesaria adaptación de la pensión de viudedad a la realidad actual}

En las últimas modificaciones de la pensión no se han tenido en cuenta los efectos de la incorporación de la mujer en el mercado laboral. Si es verdad que la Ley 40/2007 sí recogía la necesidad de este cambio para los nacidos con posterioridad a 1967 (las que en aquel momento tenían 40 ańos). No obstante, 10 años después aún no se ha incluido ninguna reforma de calado en la regulación de la viudedad. Quizás por temor a la impopularidad social que este hecho causaría, dado que muchos ciudadanos entienden que se trata de un derecho inherente a las mujeres que no han trabajado, sin darse cuenta de que el patrón a valorar se ha modificado.

Parece que, si se trata de adaptar la pensión a la realidad de la incorporación del mercado laboral, probablemente la primera opción sería su incompatibilidad con la pensión de jubilación y con el salario. De esta forma su función se dirigiría a atender a aquellos que no tienen rentas propias y que hasta ahora dependían de su cónyuge, excónyuge o pareja de hecho, que ahora ha fallecido.

De este modo, se desplazaría a la pensión de viudedad hacia el nivel asistencial, concediéndose solo a aquellas personas que hasta ahora han dependido de otra y necesitan rentas para sobrevivir. De acuerdo con los datos del INE, en el segundo cuatrimestre de 2002 había un total de 18.791,1 hombres de más de 16 años y 19.837,9 mujeres. De entre ellos son hombres inactivos 6.632,9 y 9.268,6 mujeres. En esta cifra, se excluyen tanto los que están trabajando como los que están en paro. Si bien es verdad que muchos de ellos pueden ser estudiantes, no se puede ignorar que también serán otros que no desean trabajar por diversos motivos. El hecho es que el porcentaje de mujeres es mucho más alto que los hombres.

Por lo tanto, se puede afirmar que todavía existe parte de la población que permanece en el hogar al cuidado de la familia y estas son mayoritariamente 
mujeres. De forma que si se incompatibiliza la pensión de viudedad con el salario o cualquier prestación que sustituya a este, la mayoría de quienes accederían a ella seguirían siendo beneficiarias.

Por lo tanto, hay razones para mantener que seguramente el ámbito subjetivo de la pensión de viudedad no cambiaría, esto es, seguiría siendo especialmente femenino como hasta ahora. Aunque se reduciría el número de beneficiarias a partir de cierta edad, debido a la incorporación de la mujer en el mercado laboral, posiblemente en relación con las que tienen menos de 50 ańos. Por otro lado, como es más usual que los hombres trabajen y luego accedan a la pensión de jubilación, en la mayoría de los casos no se beneficiarían de la pensión de viudedad en caso de incompatibilidad.

\subsection{La doble naturaleza de la pensión: contributiva para el causante y cuasi-asistencial para el beneficiario}

En el origen de su regulación, la pensión de viudedad era una prestación netamente contributiva a la que se accedía directamente por estar unido por nexo matrimonial con alguien que había contribuido al sistema de la Seguridad Social.

El principal requisito exigido era la contribución del causante al sistema, generalmente hombres, mientras que, por la parte del beneficiario, de forma usual beneficiaria, solo se demandaba la relación matrimonial.

Con el tiempo, el beneficio ha sufrido cambios que han provocado una suerte de asistencialización. Esta tendencia se manifiesta en las condiciones demandadas al beneficiario, mientras se mantiene los requisitos de contributividad para el causante. En diversas situaciones se solicita la acreditación de la carencia de rentas suficientes.

En primer lugar, esta tendencia se puede inferir en el reconocimiento general de la pensión de viudedad. Aunque la cuantía de la pensión de viudedad se determina aplicando un porcentaje del $52 \%$ a la base reguladora (con un anuncio de posible subida a un $65 \%$ en ciertos supuestos), es posible aumentar el importe hasta un $70 \%$ en caso de demostrar carencia de recursos e hijos a cargo.

Asimismo, donde se puede afirmar que se ha producido una total asistencialización en las notas de acceso de los beneficiarios es en el supuesto de los excónyuges y parejas de hecho. En las últimas reformas, se introdujo una limitación en el acceso de los separados, divorciados y con matrimonio nulo. Ya no se puede beneficiar directamente de la pensión, sino que para ello se exige la pérdida de pensión compensatoria en el momento del fallecimiento del causante. Esto es, será preciso demostrar que aún se dependía económicamente del excónyuge y se presumirá que, al perder esas rentas, se precisan otras en sustitución para poder subsistir. 
En idéntico sentido, cuando se extendió el derecho a la pensión de viudedad a las parejas de hecho, además de solicitar la prueba de la estabilidad notoria de la convivencia, se incluyó la exigencia de demostrar que se dependía económicamente del fallecido. Es posible acreditar la carencia de rentas de dos formas. La primera de ellas cuando los ingresos propios del beneficiario, durante el año natural anterior al fallecimiento, han sido inferiores al $50 \%$ de la suma de las rentas totales que disfrutaban el propio posible beneficiario y el causante. El límite exigido de rentas es el $25 \%$ cuando el conviviente no tenga hijos comunes con derecho a pensión de orfandad. Si hecho el cálculo, no se demuestra la falta de recursos, hay una segunda opción, que consiste en probar que sus ingresos propios son inferiores al 1,5\% del SMI vigente en el momento del hecho causante. Esta circunstancia es mejorada en el supuesto de que se tengan hijos comunes con el causante con derecho a pensión de orfandad. En este supuesto el límite se incrementa en un $0,5 \%$ del SMI por cada hijo en estas condiciones.

Es definitiva, en el caso del acceso de los convivientes a la pensión de viudedad, es preciso no solo la contribución previa a la Seguridad Social del causante, sino también la prueba de la carencia de recursos del posible beneficiario. Además, en el segundo de los supuestos señalado, habrá que seguir probando esta circunstancia durante todo el disfrute de la pensión, de modo que cuando no se cumpla, se perderá el beneficio.

En otro orden de cosas, otra cuestión que pone de manifiesto la idea de asistencialización de la pensión de viudedad es el reconocimiento generalizado del complemento a mínimos. El importe de la pensión de viudedad se calcula aplicando a la base reguladora un porcentaje del $52 \%$, que se eleva a un $70 \%$ en casos excepcionales (cuando existen cargas familiares y carencias de rentas). $\mathrm{Si}$ las bases de cotizaciones del causante son bajas, al aplicar dicho porcentaje, la cuantía final de la prestación, en muchas ocasiones, no llega a la establecida como pensión mínima. Además a este resultado vienen a contribuir otras normas: la suma de la pensión de viudedad y las pensiones de orfandad, originadas por el mismo sujeto, las cuales no pueden sumar en su conjunto más del $100 \%$ de la base reguladora (algo más en el supuesto de la aplicación del $70 \%$ de la Base Reguladora); o la posible merma del importe percibido en el supuesto de reparto de la prestación entre varios beneficiarios, cuando existan más de un cónyuge o pareja de hecho, dado que en este supuesto solo se garantiza una pensión mínima a repartir entre todos los beneficiarios.

Para reafirmar esta idea, es útil analizar el número de pensiones que se complementan a mínimos. Así, en el caso de la pensión de viudedad el porcentaje es de 33,8\% para las mujeres y 9,2\% de los hombres (MESS, agosto de 2017). En conclusión, un tercio de las pensiones de viudedad se deben complementar. Y no hay que olvidar que la financiación de dichos complementos se hace vía impuestos y que solo tienen derecho a esta mejora los beneficiarios que care- 
cen de rentas y lo acreditan. Por todo esto, se podría mantener que un tercio de las pensiones están sometidas a una suerte de asistencialización, en cuanto a que parte de su importe se recibe bajo prueba de necesidad.

Dejando a un lado lo dicho hasta el momento, las pensiones de viudedad siguen siendo prestaciones contributivas y, por tanto, se financian por cotizaciones, a excepción del complemento por mínimos. No obstante, no se puede olvidar que cada vez se han ido añadiendo más exigencias de corte asistencial para el acceso a la pensión. Con lo que surge una duda, jes posible que la naturaleza contributiva de la pensión pueda cambiar? Parece que podría ser una alternativa. De hecho, ya ha existido algún intento, proponiendo no tanto el cambio de su naturaleza como el de su financiación. Esta posibilidad no se entiende adecuada, cuestión que se analizará a continuación.

\subsection{Dos opciones de cambio ofrecidas para conseguir el equilibrio financiero}

En un futuro próximo es esperable un repunte del número de pensionistas tanto de viudedad como de jubilación. Del acceso de la mujer al mercado laboral se derivará el incremento de pensiones de jubilación, pero también el de pensiones de viudedad que irán causando las trabajadoras, aunque en menor número, dado que la esperanza de vida es mayor en la mujer. Como se sabe, esta situación provoca y va a provocar un problema importante en el ámbito de la sostenibilidad del sistema de Seguridad Social. Así, ya se están buscando soluciones a esta realidad.

En el ámbito de la pensión de viudedad caben dos opciones: buscar otros medios de financiación (a través de impuestos y no de cotizaciones), o limitar su acceso a condiciones de recursos (incompatibilizando con el disfrute de la pensión de jubilación o con el desarrollo de una actividad laboral). A continuación, se van a examinar ambas posibilidades, sobre todo vinculadas al mantenimiento del principio de contributividad.

\subsubsection{El posible cambio del tipo de financiación}

Si se centra la atención en el primer punto, enviar la financiación de la pensión de viudedad al ámbito de los impuestos, parece que esta opción podría descargar al sistema de pensiones de ciertos costes, permitiendo poner la atención del pago vía cotizaciones en la pensión de jubilación. Sin embargo, esto no parece el mejor camino.

Con independencia de que en este artículo se va a defender la opción de sustituir la pensión de viudedad por otros mecanismos de protección individual de los ciudadanos, si se mantiene esta prestación, no parece adecuado que se convierta en no contributiva mediante el traspaso de su financiación al campo de los impuestos. Esta decisión llevaría a la necesaria concesión de la prestación 
a todos los cónyuges, excónyuges y parejas de hecho. No parece adecuado demandar ningún requisito relacionado con el alta o la cotización del causante, ni diferentes condiciones para al acceso a una prestación a los distintos beneficiarios, en el supuesto de que fuese financiada por impuestos que pagan todos los ciudadanos.

Así, por ejemplo, no podría denegarse una pensión a un sujeto debido a que su cónyuge fallecido no ha contribuido lo suficiente o porque no lleve casado el tiempo requerido en el supuesto de fallecimiento por enfermedad común. Únicamente se podrían demandar condiciones relacionadas con la acreditación de insuficiencia de ingresos y, en ningún caso, de contribución.

Asimismo, como una de las finalidades principales de los impuestos es redistribuir la renta entre los ciudadanos según sus necesidades, si se incluyese la financiación de la pensión de viudedad en este ámbito, habría que concluir que rompería esta lógica cuando se concediese una pensión de viudedad a un cónyuge por el hecho de que su consorte contribuyó a la Seguridad Social.

Por otra parte, tampoco parece admisible que si las dos pensiones no contributivas, la de jubilación o por incapacidad, tienen asignada una cuantía concreta, ínfima eso sí, en el supuesto de que la pensión de viudedad fuese financiada de la misma forma, sin embargo, se calculase de acuerdo con otros parámetros como son las cotizaciones del causante. Pero, claro, si se equiparasen los importes, no tendría razón de ser la pensión de viudedad para la mayoría de sus beneficiarias mayores de 65 años, puesto que sería lo mismo recibir la pensión no contributiva de jubilación que la de viudedad.

Por todo esto, se entiende que, si se trata de mantener la pensión de viudedad en parámetros similares a los actuales, no es posible que sea financiada por impuestos. Esta opción solo se adaptaría si se cambiase profundamente las condiciones de acceso a la pensión, dejando a un lado cuestiones de corte contributivo. Y obviamente no tendría sentido limitar el derecho a los que carecen de rentas por el mero hecho de haber estado casados o viviendo en pareja y dejar a un lado al resto de los ciudadanos que igualmente precisaran de esta ayuda. Lo que, en definitiva, llevaría a la necesidad de que este tipo de derechos dejasen de ser derivados, basados en relaciones conyugales anteriores, y se convirtieran en derechos propios de todos los ciudadanos.

Antes de pasar a otro punto, parece adecuado incluir aquí una matización respecto a la pensión de orfandad. Se reconoce este derecho a los menores de 21 años o 25, si demuestran la carencia de rentas (rentas inferiores al $100 \%$ SMI anual), cuando pierden a uno de sus progenitores. La cuantía de la pensión de orfandad es solo de un $20 \%$ de la base reguladora. En el supuesto en que el causante estuviese contribuyendo por una base de cotización reducida, el 
importe de la prestación difícilmente podrá ser adecuado para dar soporte económicamente al hijo, de forma que es posible que no se respete su derecho fundamental a la educación que se recoge en la Constitución. Es por esto que, en ocasiones, la pensión de viudedad que percibe el progenitor sobreviviente puede servir como apoyo también a las necesidades de los hijos. No hay que olvidar que esta función se le asigna directamente a este beneficio cuando se eleva hasta un $70 \%$ de la base reguladora, si el beneficiario de la pensión de viudedad demuestra carencia de rentas y tener hijos a cargo.

Con todo esto lo que se quiere poner en evidencia es que quizás sí sería adecuado el traspaso de las pensiones de orfandad al ámbito no contributivo $y$, por ende, a la financiación por impuestos. De hecho, ya se ha caminado en esta dirección cuando se han modificado las condiciones contributivas exigidas al causante para el acceso a esta pensión, dado que no se demanda ningún período de carencia, ni siquiera en el caso de fallecimiento por enfermedad común.

Podría pensarse en conceder el derecho de orfandad a todos los menores de 25 años, que carezcan de rentas, sin ninguna otra vinculación con la contributividad de sus progenitores, con el fin de garantizar la igualdad de los hijos y su acceso a la educación con independencia de cualquier otra circunstancia. Obviamente esta modificación debería acompañarse de una cuantía adecuada que aportase la suficiencia precisa para estos objetivos más allá de los importes mínimos garantizados en la actualidad.

\subsubsection{La incompatibilidad de la pensión con salario y otras prestaciones sustitutivas}

Como ya se ha comentado, otra de las soluciones a barajar en aras a la sostenibilidad del sistema es mantener la pensión de viudedad en el ámbito contributivo, pero solo dejar acceder a ella a quienes no estén trabajando. Si se incompatibiliza la pensión con el salario, consecuentemente se deberá hacer los mismo con cualquier prestación que sustituya a la retribución como, por ejemplo, con la pensión de jubilación.

Esta opción reduciría el ámbito subjetivo de la pensión de viudedad, pero tendría un efecto vulnerador del principio de contributividad.

El principio de contributividad básicamente significa que quien contribuyó a la Seguridad Social a través de sus cotizaciones, debe recibir una prestación en el futuro si sufre una situación de necesidad. Así, dos personas, que contribuyen igual, deberían recibir una protección idéntica. Esto, en cierto modo, se rompe con la lógica de la pensión de viudedad, ya que un sujeto que cause este beneficio puede haber percibido una pensión de jubilación y, posteriormente, su cónyuge disfrutar de la pensión de viudedad. Es decir, habría generado dos pensio- 
nes contributivas. Sin embargo, un sujeto que contribuye de igual forma y no esté casado o viva en pareja, solo podrá causar una pensión, en su caso, la suya propia de jubilación.

Si se incompatibiliza pensión viudedad-salario/jubilación, la brecha será aún mayor. Con independencia de que se cotice en igual medida, solo aquellos que tengan un cónyuge/pareja de hecho que no trabaje, causarían dos pensiones (la propia y, en su caso, la de viudedad) frente a la situación general del resto de los cotizantes.

Hay razones para defender que la lógica contributiva se rompe cuando la razón para causar o no más de una pensión con las mismas cotizaciones, se base en cuestiones de la vida privada de cada sujeto e incluso de opciones vitales como quedarse en casa al cuidado del hogar.

Por otro lado, como ya se ha señalado, del análisis de los datos del número de inactivos, lo cual no hay que confundir con parados, hay que concluir que hay un mayor número de mujeres en esta situación, lo cual lleva a intuir que, en muchos casos se debe a la opción de ellas de mantenerse en el hogar al cuidado de su familia.

De modo que la decisión de incompatibilizar la pensión de viudedad con salario/jubilación lleva por un lado a vulnerar en mayor medida el principio de contributividad y, por otro, a perpetuar roles sociales que se tuvieron en cuenta al regular la pensión de viudedad hace más de 50 años, esto es, el reparto clásico de las tareas entre el hombre trabajador y la mujer cuidadora (Rodríguez, 2006: 22).

De acuerdo con lo señalado, si se opta por mantener la pensión de viudedad, desde aquí se apuesta por no modificar el requisito de la compatibilidad con el salario y prestaciones que le sustituyan. De este modo, por una parte, aunque si se mantiene cierta ruptura con el principio de contributividad, la brecha es menor que si se reduce el ámbito de los causantes del beneficio y, por otra, no se perpetuaran roles propios de décadas anteriores.

\subsection{Hacia el necesario reconocimiento de derechos propios a los ciudadanos}

Después de todo lo señalado, hay que concluir que la principal reivindicación de este trabajo consiste en entender que la pensión de viudedad ha cumplido la función que le fue designada hace 50 años, pero que ahora ha dejado de tener sentido mantenerla.

Antes de entrar en esta propuesta, es vital hacer una matización. Se considera fundamental el mantenimiento de la pensión de viudedad para todas aquellas mujeres, que debido al reparto de roles entre hombres y mujeres hace unas décadas, no desarrollaron actividad laboral fuera de casa y, por tanto, no 
pueden acceder a la pensión de jubilación por sí mismas. Respecto a este colectivo parece que lo más adecuado es mantener la pensión de viudedad tal y como está configurada hoy en día, sin incluir ningún cambio en la fuente de financiación.

No obstante, de acuerdo con la evolución social y la incorporación de la mujer al mercado laboral no existen fundamentos para mantener una pensión con esta configuración para las mujeres que ya no han estado sometidas a este reparto de roles. No parece adecuado que se conceda un beneficio por cuestiones relativas a las elecciones personales de los ciudadanos: casarse, unirse de hecho o no decantarse por ninguna de ellas. No puede ligarse a este hecho tan personal que una persona acceda a unas rentas para garantizar su subsistencia.

El problema fundamental en este punto es establecer el momento de inflexión en el que se ha producido este cambio social y, por tanto, determinar desde cuándo hay que introducir las modificaciones. De acuerdo con lo establecido en la Ley 40/2007 podría elegirse el año 1967. Es decir, que la modificación se introduciría para los ciudadanos con menos de 50 años.

Como se ha puesto de manifiesto, existe una tendencia hacia la asistencialización de los requisitos exigidos a los beneficiarios de la pensión de viudedad. Cada vez se vincula más el acceso a la pensión de viudedad a elementos de prueba de carencia de rentas, por ejemplo, en el caso de las parejas de hecho o de los excónyuges. Si finalmente solo se van a mantener los requisitos de contributividad en relación con el causante, parece que lo adecuado es replantearse la naturaleza de la pensión de viudedad. Una vez hecho esto, parece que ya no queda justificación alguna para conceder el beneficio por razón de la vinculación de un sujeto a otro, sino que lo adecuado será la atención por ser una persona en situación de necesidad.

Así, los derechos de protección social deben ser propios, por el hecho de ser un ciudadano, no derivados y unidos a requisitos de relaciones matrimoniales o de convivencia. Cuando una persona demuestra su carencia de rentas, debería poder acceder a una renta suficiente para sobrevivir, independientemente de cualquier otra circunstancia personal. Aunque seguramente, como ocurre en el ámbito de las pensiones no contributivas de jubilación, una gran mayoría de las personas que accediesen serían mujeres.

No obstante, es verdad que si se tiene una unidad familiar y se pierde la renta que uno de los sustentadores aporta, es preciso adaptarse a la nueva situación, lo que precisa de un cierto tiempo de transición, sobre todo en el supuesto de que el otro no tenga una retribución propia. Es por esto por lo que parece adecuado regular un subsidio de viudedad por dos años, en la misma forma que existe en la actualidad para aquellos que no demuestren que la duración de su 
matrimonio es superior a un ańo (o 2 en caso de convivencia y el nexo matrimonial) o tienen un hijo común, cuando el causante falleció por enfermedad común que ya se conocía en el momento que formalizaron la unión. Con esta prestación se concedería un tiempo a los cónyuges o parejas para rehacer su situación económica.

En todo caso, debe entenderse que esta protección no debe vincularse al ámbito asistencial de las comunidades autónomas, sino a un derecho subjetivo reconocido en el ámbito de la Seguridad Social, tal y como se regula en otros países como Francia o Reino Unido. Incluso en este último sistema se ha decidido reconducir la protección en el marco de los derechos propios, eliminando los derivados (la pensión de viudedad), creando una nueva renta para todos ciudadanos que carecen de recursos vinculada a la protección por desempleo (Blázquez, 2017: 56 y ss.).

Otra opción adecuada podría ser la que ofrece el derecho francés, donde al fallecimiento del cónyuge se aumenta la cuantía de la pensión de jubilación. Así, en estos momentos solo aproximadamente un cuarto de la viudedad se recibe como pensión de esta naturaleza, mientras que el resto se percibe como complemento de la jubilación, siendo las mujeres más mayores las que mantienen la primera opción (Collin, 2016).

Por otro lado, como ya se ha mencionado, hay que facilitar la continuación de la vida familiar en el supuesto de los progenitores que quedan solos a cargo de sus hijos al fallecimiento del otro. Es por esto por lo que en este contexto parece que la única solución es reconocer pensiones de orfandad económicamente suficientes para el sostenimiento de los hijos, lo cual hay razones para defender como factible en el supuesto que no sea preciso abonar el porcentaje seńalado para la pensión de viudedad y se pueda dedicar a proteger a los hijos en el ámbito contributivo o, aún mejor, reconocer el derecho como una prestación universal para todos los hijos.

\section{Bibliografía}

Blázquez Agudo, Eva María (2017): «El incentivo a la búsqueda de trabajo en el derecho inglés», Trabajo y Derecho, 30.

Collin, Chistel (2016): «La part de la réversion dans la retraite des femmes diminue au fil des générations», Études et Résultats, 951.http://drees.solidarites-sante.gouv.fr/etudeset-statistiques/publications/etudes-et-resultats/article/la-part-de-la-reversion-dans-laretraite-des-femmes-diminue-au-fil-des (último acceso 21 de septiembre de 2017).

Durán Heras, María Ángeles (2006): «Dependientes y cuidadores: el desafío de los próximos años», Revista del Ministerio de Trabajo y Asuntos Sociales, 60. 
INE (2010): Encuesta de Empleo del Tiempo, http://www.ine.es/dyngs/INEbase/ es/operacion.htm?c=Estadistica_C\&cid $=1254736176829 \&$ menu $=$ resultados $\&$ id $\mathrm{p}=1254735976595$ (último acceso 21 de septiembre de 2017).

Encuesta de Población Activa (2011): Módulo sobre conciliación entre la vida laboral y la familiar del año 2010.

IMSERSO (1995): Cuidados en la vejez. El apoyo informal, Ministerio de Asuntos Sociales, Madrid.

MESS (2017): Estadísticas, http://www.seg-social.es/Internet_1/Estadistica/Est/ Pensiones_y_pensionistas/index.htm (último acceso 21 de septiembre de 2017).

Rodríguez Rodríguez, Pilar (2006): El sistema de servicios sociales español y las necesidades derivadas de la atención a la dependencia, Fundación Alternativas, Documento de Trabajo 87 . 